\title{
Chinese nomogram to predict probability of positive initial prostate biopsy: a study in Taiwan region
}

\author{
Shu-Chun Kuo ${ }^{1,2}$, Shun-Hsing Hung ${ }^{3}$, Hsien-Yi Wang ${ }^{4,5}$, Chih-Chiang Chien ${ }^{4,6}$, Chin-Li Lu ${ }^{7}$, Hung-Jung Lin ${ }^{8,9}$, \\ How-Ran Guo ${ }^{10}$, Jian-Fang Zou ${ }^{11}$, Chian-Shiung Lin $^{9,12}$ and Chien-Cheng Huang, ${ }^{8,10,13}$
}

Several nomograms for prostate cancer detection have recently been developed. Because the incidence of prostate cancer is lower in Chinese men, nomograms based on other populations cannot be directly applied to Chinese men. We, therefore, developed a model for predicting the probability of a positive initial prostate biopsy using clinical and laboratory data from a Chinese male population. Data were collected from 893 Chinese male referrals, 697 in the derivation set and 196 in the external validation set, who underwent initial prostate biopsies as individual screening. We analyzed age, prostate volume, total prostate-specific antigen (PSA), PSA density (PSAD), digital rectal examinations (DRE) and transrectal ultrasound (TRUS) echogenicity. Logistic regression analysis estimated odds ratio, $95 \%$ confidence intervals and $\boldsymbol{P}$ values. Independent predictors of a positive biopsy result included advanced age, small prostate volume, elevated total PSA, abnormal digital rectal examination, and hyperechoic or hypoechoic TRUS echogenicity. We developed a predictive nomogram for an initial positive biopsy using these variables. The area under the receiver-operating characteristic curve for the model was $\mathbf{8 8 . 8 \%}$, which was greater than that of the prediction based on total PSA alone (area under the receiver-operating characteristic curve $74.7 \%$ ). If externally validated, the predictive probability was 0.827 and the accuracy rate was $78.1 \%$, respectively. Incorporating clinical and laboratory data into a prebiopsy nomogram improved the prediction of prostate cancer compared with predictions based solely on the individual factors.

Asian Journal of Andrology (2013) 15, 780-784; doi:10.1038/aja.2013.100; published online 14 October 2013

Keywords: biopsy; Chinese; nomogram; prediction; prostate cancer

\section{INTRODUCTION}

In Taiwan region, a predominantly Chinese population, the incidence of prostate cancer was not in the top 10 in 1998; however, in 2008 , its incidence rose to the fifth and it became the seventh most frequent cause of cancer death. ${ }^{1}$ In every year since 1998, over 2000 patients have been diagnosed with prostate cancer and over 750 patients in all have died from it. Unfortunately, in spite of medical advancement, the number of patients newly diagnosed with prostate cancer and the mortality rate of the disease continue to rise. ${ }^{1}$ In addition, metastatic prostate cancer is significantly higher in Taiwan region's Chinese population than in the general American population $(32.7 \%$ vs. $5 \%) .^{2}$

Early detection and intervention of prostate cancer are effective. ${ }^{3}$ Widespread use of serum prostate-specific antigen (PSA) screening has increased the detection of early prostate cancer. ${ }^{4}$ However, serum PSA levels increase, not only in patients with prostate cancer, but also in various nonmalignant conditions of the prostate. ${ }^{4}$ Discriminating prostate cancer from benign prostatic disease is difficult; therefore, the number of unnecessary prostate biopsies has increased with the increase in PSA screening. ${ }^{4}$

Although invasive and costly prostate biopsies provide a definitive diagnosis, they should be avoided in men with a low probability of disease because of the possible complication and associated pain. ${ }^{4,5}$ In addition, waiting for the biopsy leads to patient anxiety. About $55 \%$ of patients felt anxiety when waiting for prostate biopsy, and $6 \%$ experienced high levels of anxiety. ${ }^{6}$ Efforts to develop predictive models for prostate cancer using clinical, laboratory and ultrasound parameters have been directed to improve the rates of prostate cancer detection and to reduce patient anxiety. ${ }^{6-11}$

Nomograms are widely used for cancer prognosis, primarily because they reduce statistical predictive models into a single numerical estimate, tailored to the profile of an individual patient, of the probability of an event, such as death or recurrence. ${ }^{12}$ User-friendly graphical interfaces for generating these estimates facilitate using nomograms to inform clinical decision making. ${ }^{12}$ In 2003, a nomogram using age, prostate-specific antigen density (PSAD), digital rectal

${ }^{1}$ Department of Ophthalmology, Chi-Mei Medical Center, Tainan 710; ${ }^{2}$ Department of Optometry, Chung Hwa University of Medical Technology, Tainan $710 ;{ }^{3}$ Division of Urology, Departments of Surgery, Chi-Mei Medical Center, Tainan 710; ${ }^{4}$ Department of Nephrology, Chi-Mei Medical Center, Tainan $710 ;{ }^{5}$ Department of Sport Management, College of Leisure and Recreation Management, Chia Nan University of Pharmacy and Science, Tainan 710; ${ }^{6}$ Department of Food Nutrition, Chung Hwa University of Medical Technology, Tainan 710; ${ }^{7}$ Chia-Yi Christian Hospital, Chia-Yi 600; ${ }^{8}$ Department of Emergency Medicine, Chi-Mei Medical Center, Tainan 710; ${ }^{2}$ Department of Biotechnology, Southern Taiwan University of Science and Technology, Tainan 710; ${ }^{10}$ Department of Environmental and Occupational Health, Medical College, National Cheng Kung University, Tainan 710; ${ }^{11}$ Clinical Division of Occupational Medicine, Institute of Occupational Health and Occupational Medicine, Academy of Medical Science, Jinan 250000 , China; ${ }^{12}$ Divisions of Urology, Department of Surgery, Chi-Mei Medical Center, Liouying 736 and ${ }^{13}$ Department of Child Care and Education, Southern Taiwan University of Science and Technology, Tainan 710

Correspondence: Dr CS Lin (kkslin@yahoo.com.tw) and Dr CC Huang (chienchenghuang@yahoo.com.tw)

Received: 8 March 2013; Revised: 22 June 2013; Accepted: 12 July 2013; Published online: 14 October 2013 
examination (DRE) and transrectal ultrasound (TRUS) in patients with intermediate PSA levels was developed to predict the presence of prostate cancer. ${ }^{13}$ Two others were developed for predicting the probability of a positive prostate biopsy. ${ }^{14}$ One study that compared three commonly used nomograms (the CRC, SWOP-PRI and Montreal) concluded that the Montreal nomogram was the best choice. ${ }^{15}$ A nomogram for specifically for Japanese patients was also developed. ${ }^{4}$ However, because prostate cancer is thought to differ epidemiologically and biologically between Western, Japanese and Chinese populations, ${ }^{16}$ nomograms developed for other populations cannot be directly applied to the Chinese population in Taiwan region, which has a lower incidence of prostate cancer than non-Chinese populations do. Our aim was to construct a nomogram to predict the probability of a positive prostate biopsy using data from a Chinese population.

\section{MATERIALS AND METHODS}

\section{Study population}

Between January 2009 and December 2011, data were retrospectively collected for the purposes of clinical care from 893 referred patients who underwent an initial TRUS-guided prostate biopsy at Chi Mei Young-Kang Medical Center and Chi Mei Liou-Ying Hospital. Patients were referred for initial TRUS-guided prostate biopsy when he met any of the following criteria: total PSA $>4.0 \mathrm{ng} \mathrm{ml}^{-1}$, PSAD $>0.15 \mathrm{ng} \mathrm{ml}^{-1} \mathrm{~cm}^{-3}$, suspect cancer on DRE, and hyperechoic or hypoechoic TRUS echogenicity. The studied hospital's institutional review board approved the protocol. The patients were divided into derivation and external validation sets. The derivation set consisting of 697 patients from Chi Mei Young-Kang Medical Center, was for building the nomogram. The external validation set consisting of 196 patients from Chi Mei Liou-Ying Hospital, was for testing the nomogram. The mean PSA level before the biopsy was $28.3 \pm 33.8 \mathrm{ng} \mathrm{ml}^{-1}$ (range: $0.11-100.0 \mathrm{ng} \mathrm{ml}^{-1}$ ). One member of the team of urologists did a DRE on all patients before the TRUS test, and the results were classified as normal or suspect cancer. Patients with prostatic nodules and indurations on the DRE were suspected of having cancer.

\section{Serum total PSA assay}

A single blood sample drawn before the DRE was immediately centrifuged to separate the serum and then stored at $-20^{\circ} \mathrm{C}$. A total PSA assay was done (Chemiluminescent Microparticle Immunoassay Architect System; Abbott Ireland Diagnostic Division, Sligo, Ireland).

\section{TRUS-guided systematic biopsy}

A TRUS-guided systematic biopsy of the prostate was done using surgical ultrasonography (Biplane transducer 8808 mode $(10 \mathrm{MHz})$; BK Medical, Herlev, Denmark) under local anesthesia with patients placed in the left-lateral decubitus position. The probe was inserted transrectally, and the prostate was scanned simultaneously in the transverse and sagittal planes. The prostate volume was calculated using the formula for a prolate ellipsoid (length $\times$ width $\times$ height $\times 0.523$ ). An automatic biopsy gun and an 18-gauge needle with TRUS guidance were used. Biopsies were performed by urologists and the numbers of biopsies from each patient were all $\geqslant 10$ specimens. If there were obvious hypoechoic prostate lesions on the ultrasound images, five specimens were taken from those areas, while the remaining specimens were obtained randomly from the peripheral and transitional zones of each lobe. If no hypoechoic prostate lesion was observed on TRUS imaging, all specimens were randomly obtained from the peripheral and transitional zones of each lobe equally.

\section{Statistical analysis}

Mean and standard deviation were calculated for age, prostate volume, total PSA and PSAD, and count and percentage were calculated for DRE findings and TRUS echogenicity. Considering the ease for clinical practice, continuous factors were categorized into quartiles for the following analyses.

A prediction model was conducted based on the data collected from Chi Mei Yung-Kang Medical Center. Both univariate and multivariate logistic regression analysis were used to examine the association between predictive variables and biopsy outcomes. Crude or adjusted odds ratios and $95 \%$ confidence intervals (CI) were calculated. Using a backward model-selection procedure, the final model for prediction was conducted. Model fitness and interpretability were assessed using the Hosmer-Lemeshow and the Nagelkerke $r^{2}$ tests, respectively. Receiver-operating characteristic curves and C-statistics were used to assess the model's ability to discriminate. The model was also externally validated. Using the prediction model for the data from the patients from Chi Mei Liou-Ying Hospital, C-statistics for the probability derived from the model and for each individual factor were calculated and compared.

Based on the final model, the estimated probability of a positive biopsy could be calculated, a nomogram was accordingly developed to use as a clinical tool. In brief, the development method was that the regression coefficients representing the strengths of correlation were proportionately transferred to the distances on the graph, on which they can be linked to corresponding points and summed to a total. The estimated probability of a positive biopsy thus can thus be determined. SPSS 17.0.1 for Windows (SPSS Inc., Chicago, IL, USA) and Microsoft Office Excel 2007 (Microsoft, Redmond, WA, USA) were used for all statistical analyses.

\section{RESULTS}

\section{Patient data}

The mean standard deviation and median range of age, prostate volume, total PSA and PSAD were shown in Table 1. The comparison

Table 1 Clinical characteristics of 697 patients in the derivation set and 196 patients in the validation set with initial biopsy results (mean \pm s.d., median and range in parenthesis)

\begin{tabular}{|c|c|c|c|c|}
\hline \multirow[t]{3}{*}{ Variable } & Derivation set & \multirow{3}{*}{$\begin{array}{l}\text { Positive biopsy } \\
\qquad(\mathrm{n}=240)\end{array}$} & \multirow{3}{*}{$\begin{array}{l}\text { Negative biopsy } \\
\qquad(n=457)\end{array}$} & Validation set \\
\hline & Total patients & & & Total patients \\
\hline & $(n=697)$ & & & $(n=196)$ \\
\hline Age (year) & $68.5 \pm 10.6(70.0,29-96)$ & $72.5 \pm 9.2(73.0,29-95)$ & $66.4 \pm 10.6(68.0,31-96)$ & $69.0 \pm 10.6(70.0,38-89)$ \\
\hline Prostate volume $\left(\mathrm{cm}^{3}\right)$ & $43.3 \pm 22.1(38.9,6.0-233.0)$ & $41.6 \pm 19.2(36.4,8.2-115.8)$ & $44.2 \pm 23.5(39.7,6.0-233.0)$ & $41.6 \pm 26.2(34.9,9.1-203.8)$ \\
\hline Total PSA (ng ml-1) & $28.3 \pm 33.8(11.2,0.1-100.0)$ & $56.2 \pm 39.8(50.2,0.6-100.0)$ & $13.7 \pm 17.0(8.5,0.1-100.0)$ & $20.1 \pm 21.1(11.5,0.25-100.0)$ \\
\hline PSAD (ng ml cm ${ }^{-3}$ ) & $0.7 \pm 1.0(0.3,0-12.1)$ & $1.5 \pm 1.3(1.3,0-12.1)$ & $0.3 \pm 0.4(0.2,0-4.3)$ & $0.6 \pm 0.8(0.3,0-4.9)$ \\
\hline
\end{tabular}

Abbreviations: PSA, prostate-specific antigen; PSAD, prostate-specific antigen density. 
Table 2 Univariate analysis evaluating risk of positive biopsy in the derivation set

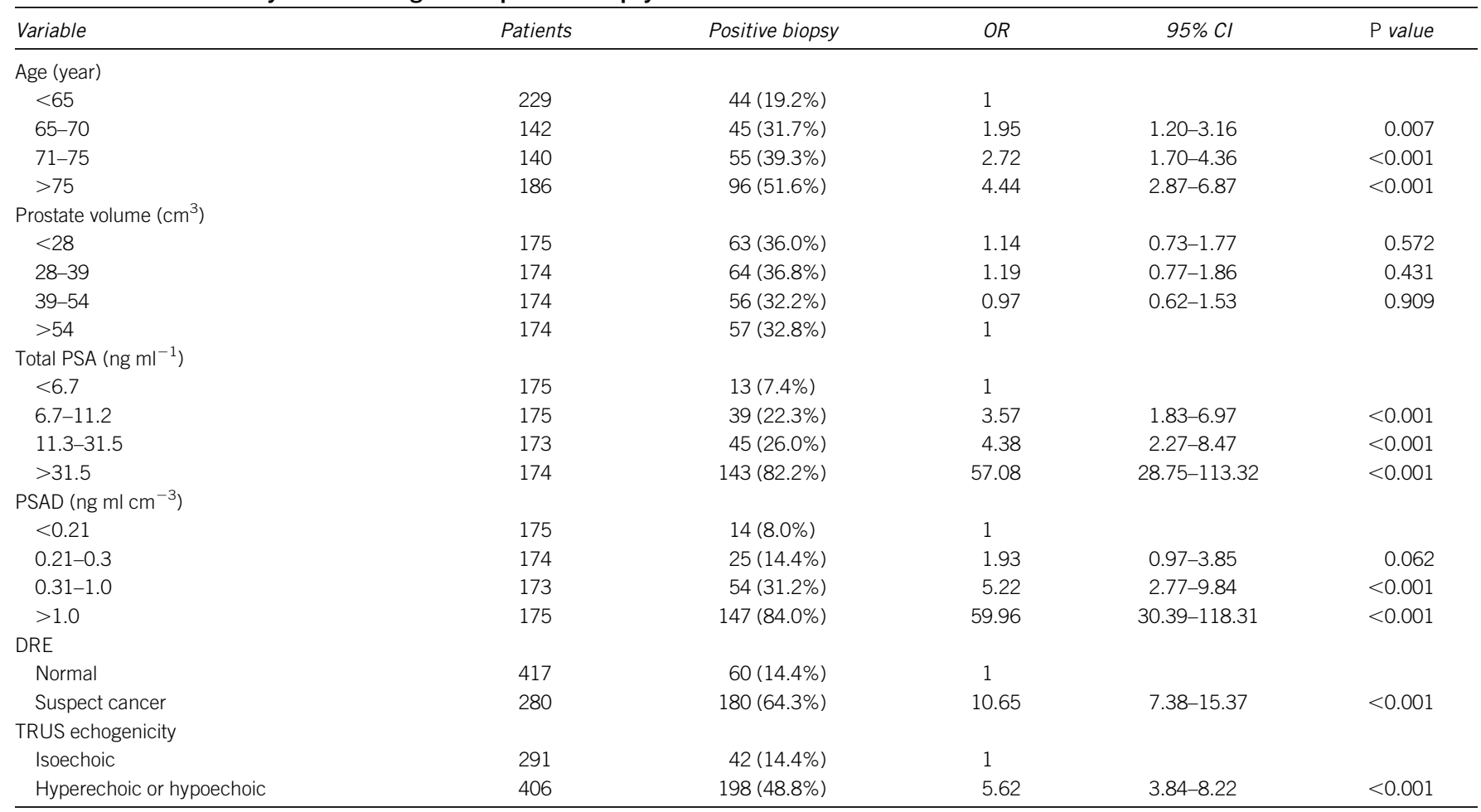

Abbreviations: $\mathrm{Cl}$, confidence interval; DRE, digital rectal examination; OR, odds ratio; PSA, prostate-specific antigen; PSAD, prostate-specific antigen density; TRUS, transrectal ultrasound.

of percentage of positive biopsy, age, prostate volume, total PSA, PSAD, DRE and TRUS echogenicity between derivation set and external validation set was showed in Table 1. The DRE findings were classified as suspect cancer in $280(40.2 \%)$ patients (Table 2). The TRUS echogenicity findings were hypoechoic or hyperechoic in 406 (58.2\%) and isoechoic in 291 (41.8\%).

\section{Biopsy results}

Prostate cancer was detected in 240 (34.4\%) of the derivation set biopsy specimens. Table 2 shows the patient distribution within each variable and the results of the univariate analysis that evaluated the risk associated with biopsies.

\section{Nomogram development}

Univariate logistic regression analysis indicated that age, total PSA, DRE and TRUS echogenicity are the four significant predictors for a positive prostate biopsy (Table 2). A stepwise multivariate logistic regression analysis showed that the most significant of the five risk factors for detecting prostate cancer are total PSA, DRE and TRUS echogenicity (Table 3). Independent analyses using forward and backward stepwise procedures yielded identical results. A nomogram was developed using the five independent risk factors to diagnose prostate cancer (Figure 1). For each of the five categories, an individual accumulated a number of points that were summed to calculate the overall likelihood of a positive biopsy, as described elsewhere. ${ }^{17}$ For example, for a 72-year-old man with a PSA level of $8.5 \mathrm{ng} \mathrm{ml}^{-1}$, a prostate volume of $20 \mathrm{~cm}^{3}$, and normal DRE and TRUS hyperechoic findings, according to the model, his risk of a positive biopsy would be $45 \%$, not the $25 \%$ usually indicated by an intermediate PSA level of 4 $10 \mathrm{ng} \mathrm{ml}^{-1}$. Receiver-operating characteristic analysis indicated that
Table 3 Multivariate analysis evaluating risk of positive biopsy in the derivation set

\begin{tabular}{|c|c|c|c|c|c|}
\hline Variable & $O R$ & $95 \% \mathrm{Cl}$ & P value & Beta & \\
\hline \multicolumn{6}{|l|}{ Age (year) } \\
\hline$<65$ & 1 & & & & \\
\hline $65-70$ & 1.66 & $0.87-3.17$ & 0.127 & 0.51 & \\
\hline $71-75$ & 1.95 & $1.04-3.68$ & 0.038 & 0.67 & \\
\hline$>75$ & 2.27 & $1.26-4.09$ & 0.007 & 0.82 & 0.82 \\
\hline \multicolumn{6}{|l|}{ Prostate volume $\left(\mathrm{cm}^{3}\right)$} \\
\hline$<28$ & 4.04 & $2.09-7.78$ & $<0.001$ & 1.40 & 1.4 \\
\hline 28-39 & 2.19 & $1.18-4.08$ & 0.013 & 0.78 & \\
\hline $39-54$ & 1.30 & $0.67-2.43$ & 0.411 & 0.26 & \\
\hline$>54$ & 1 & & & & \\
\hline \multicolumn{6}{|l|}{ Total PSA (ng ml ${ }^{-1}$ ) } \\
\hline$<6.7$ & 1 & & & & \\
\hline $6.7-11.2$ & 7.06 & 3.33-14.98 & $<0.001$ & 1.95 & \\
\hline $11.3-31.5$ & 4.99 & $2.40-10.36$ & $<0.001$ & 1.61 & \\
\hline$>31.5$ & 39.65 & $18.10-86.87$ & $<0.001$ & 3.68 & 3.68 \\
\hline \multicolumn{6}{|l|}{ DRE } \\
\hline Normal & 1 & & & & \\
\hline Suspect cancer & 3.48 & $2.17-5.58$ & $<0.001$ & 1.25 & 1.25 \\
\hline \multicolumn{6}{|l|}{ TRUS echogenicity } \\
\hline Isoechoic & 1 & & & & \\
\hline $\begin{array}{l}\text { Hyperechoic or hypoechoic } \\
\text { Constant }\end{array}$ & 3.46 & $2.11-5.68$ & $<0.001$ & $\begin{array}{c}1.24 \\
-5.141\end{array}$ & 1.24 \\
\hline
\end{tabular}

Abbreviations: $\mathrm{Cl}$, confidence interval; DRE, digital rectal examination; OR, odds ratio; PSA, prostate-specific antigen; PSAD, prostate-specific antigen density; TRUS, transrectal ultrasound.

Hosmer-Lemeshow test, $P=0.142$; Nagelkerke, $r^{2}=0.557$. 


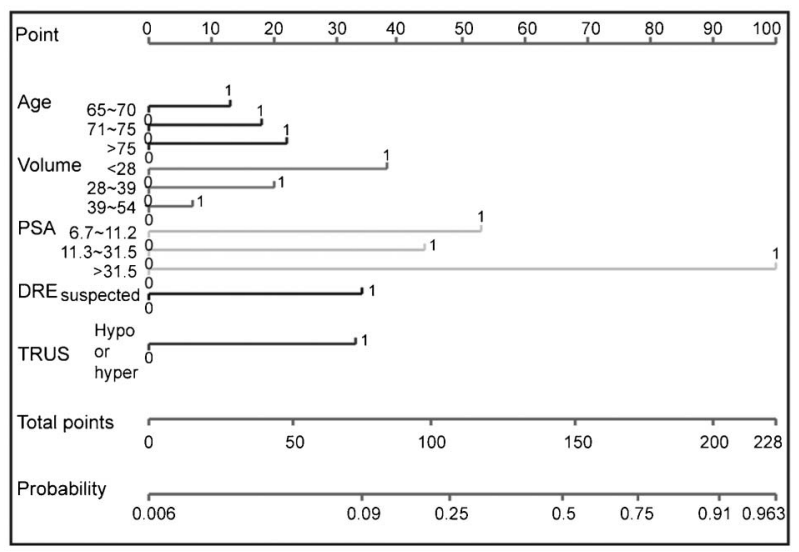

Figure 1 Chinese nomogram for detecting prostate cancer by initial biopsy. Locate age, prostate volume, total PSA, DRE and TRUS echogenicity for individual patients. Draw a line upward to number of points in each category. Sum the points and draw a line downward to find the risk of a positive biopsy. DRE, digital rectal examinations; PSA, prostate-specific antigen; TRUS, transrecta ultrasound.

Table 4 Comparison of nomogram results and other predictors using ROC analysis

\begin{tabular}{lc}
\hline Model & Area under the curve $(95 \% \mathrm{Cl})$ \\
\hline Present nomogram & $0.888(0.861-0.914)$ \\
Age (year) & $0.671(0.629-0.712)$ \\
Prostate volume $\left(\mathrm{cm}^{3}\right)$ & $0.474(0.429-0.519)$ \\
Total PSA $\left(\mathrm{ng} \mathrm{ml}^{-1}\right)$ & $0.747(0.714-0.780)$ \\
PSAD $\left(\mathrm{ng} \mathrm{ml}^{-1} \mathrm{~cm}^{-3}\right)$ & $0.772(0.740-0.805)$ \\
\hline
\end{tabular}

Abbreviations: $\mathrm{Cl}$, confidence interval; PSA, prostate-specific antigen; PSAD, prostate-specific antigen density; ROC, receiver-operating characteristic.

the accuracy of the predicted probability for the model was $88.8 \%$ compared with $74.7 \%$ when using total PSA alone (Table 4). The accuracy of this model was also determined using the external validation set: predictive probability $=0.827$; accuracy $=78.1 \%$. We also compared our nomogram to the work by Tang et al. ${ }^{18}$ (Table 5).

\section{DISCUSSION}

In the present study, we developed a predictive model that incorporates clinical and laboratory data from general practice to detect prostate cancer in a Chinese population. The significant independent covariates for detecting cancer in the present study were age, prostate volume, total PSA, DRE findings and TRUS echogenicity. Using these risk factors, we constructed a Chinese nomogram that provides more precise risk-analysis information for individual Chinese patients. This predictive nomogram for the Chinese population might aid physicians and patients in electing to undergo a prostate biopsy, the definitive diagnostic tool. It not only may facilitate early prostate cancer diagnosis, but also may help diminish unnecessary prostate biopsies and reduce patient anxiety.

Tang et al. ${ }^{18}$ also proposed a nomogram to be used for decide whether to perform a biopsy in Chinese patients. Comparing to the work by them, our nomogram enrolled more patients (893 vs. 535) and showed more accurate (external validation $v s$. internal validation) and better area under the curve (AUC; 88.8\% vs. 84.8\%) and increase AUC vs. PSA alone (14.1\% vs. 5.1\%) (Table 5). We also compared our model with other Western and Japanese nomograms in Table 5.

Several models-nomograms, risk groupings, artificial neural networks, probability tables, and logistic regression analysis-have been developed to help predict a positive prostate biopsy in men being evaluated for prostate cancer. ${ }^{4,22-24}$ Nomograms, artificial neural networks and logistic regression analysis improved the accuracy of prediction compared with the individual factors alone.

The statistical definition of a nomogram is a graphical representation of a mathematical formula or algorithm that incorporates several predictors modeled as continuous variables to predict a particular end point based on traditional statistical methods, such as multivariable logistic regression and Cox proportional hazards analysis. ${ }^{25}$ Nomograms use continuous scales to calculate the continuous probability of a particular outcome. This obviates the effect of spectrum bias that might be operational when predictors are stratified. Spectrum bias consists of a forced central effect applied to the entire range of observations that decrease within the limits of a given category.

Nomograms are perfect examples of a predictive application that allows a graphical representation of variable interactions and a depiction of their combined effects. Nomograms provide superior individualized disease-related risk estimations that facilitate patient management-related decisions. Nomograms are the most accurate and have the best discriminating characteristics with of the currently available tools for predicting outcomes in patients with prostate cancer. $^{25}$

This study has several limitations. First, the data were collected from a retrospective chart review. These clinical presentations or records may not have been completely documented. Second, this was a singlecenter study. Findings from our database may not be generalizable to cohorts in other Chinese populations. Third, many more nomograms, as well as improvements to existing nomograms, are needed. For example, none of the nomograms predicts with perfect accuracy. Novel biomarkers, larger data sets, better data collection methods and more sophisticated modeling procedures are needed to improve

Table 5 Predictive accuracy of two Chinese nomograms (Tang et al. ${ }^{18}$ and our model) and other Western nomograms for predicting probability of positive initial prostate biopsy

\begin{tabular}{|c|c|c|c|c|c|c|c|c|}
\hline Study & Patients (n) & Cancer rate (\%) & Validation & Mean age (year) & $\begin{array}{c}\text { Median PSA } \\
\quad\left(n g m l^{-1}\right)\end{array}$ & AUC for model (\%) & $A \cup C$ for $P S A(\%)$ & $\begin{array}{l}\text { Increase in AUC } \\
\text { vs. PSA alone (\%) }\end{array}$ \\
\hline Tang et al. ${ }^{18}$ & 535 & 44.8 & Internal & 72 & 17.8 & 84.8 & 79.7 & 5.1 \\
\hline Our model & 893 & 34.4 & External & 68.5 & 11.2 & 88.8 & 74.7 & 14.1 \\
\hline $\mathrm{PCPT}^{19}$ & 5519 & 21.9 & Internal & $\geqslant 55$ & 1.5 & 70.2 & 67.8 & 2.4 \\
\hline Hernandez et al. ${ }^{20}$ & 1108 & 35.6 & NA & 63 & 5.4 & 66.7 & 61.9 & 4.8 \\
\hline Suzuki et al. ${ }^{4}$ & 834 & 28.9 & NA & 70 & 13.1 & 81.8 & 69.8 & 12.0 \\
\hline Park et al. ${ }^{21}$ & 602 & 28.6 & NA & 66 & 6.77 & 72 & 66 & 6 \\
\hline Garzotto et al. ${ }^{13}$ & 1239 & 24.3 & Internal & 66 & 5.10 & 73 & 62 & 11 \\
\hline
\end{tabular}

Abbreviations: AUC, area under the curve; NA, not available; PCPT, Prostate Cancer Prevention Trial; PSA, prostate-specific antigen. 
predictive accuracy. Fourth, better accuracy might be achieved by modeling physician or hospital-specific data, or both, for patients being treated by that physician or at that hospital. Fifth, the current study enrolled the patients with suspected prostate cancer whose total PSA and positive DRE would be higher than the study enrolling patients for routine screening.

\section{AUTHOR CONTRIBUTIONS}

CSL takes responsibility for the paper as a whole. SCK, CSL and CCH conceived the study concept and design, acquired data, performed statistical analysis, analyzed and interpreted the data, wrote the manuscript and revised the manuscript. CLL performed statistical analysis, analyzed and interpreted data, and reviewed and edited the manuscript. HYW, CCC, HJL, HRG and JFZ reviewed and edited the manuscript. SHH acquired, analyzed and interpreted data. All authors read and approved the final manuscript.

\section{COMPETING FINANCIAL INTERESTS}

All authors declare no competing financial interests.

\section{ACKNOWLEDGMENTS}

This study was supported in part by grants CLFHR9947 and CMFHR10013 from the Chi-Mei Medical Center. We thank Bill Franke for his invaluable advice and editorial assistance.

1 Taiwan Cancer Registry. Available from: http://crs.cph.ntu.edu.tw/main.php?Page= A5 and http://tcr.cph.ntu.edu.tw/uploadimages/Top\%2010\%20cancer\%20in\% 20Taiwan\%202008.pdf (accessed 22 January 2013).

2 National Cancer Institute: SEER Cancer Statistics Review. Available from: http:// seer.cancer.gov/csr/1975_2002/results_merged/sect_23_prostate.pdf (accessed 22 January 2013).

3 Bill-Axelson A, Holmberg L, Ruutu M, Häggman M, Andersson SO et al. Radical prostatectomy versus watchful waiting in early prostate cancer. N Engl J Med 2005; 352: $1977-84$.

4 Suzuki H, Komiya A, Kamiya N, Imamoto T, Kawamura K et al. Development of a nomogram to predict probability of positive initial prostate biopsy among Japanese patients. Urology 2006; 67: 131-6.

5 Rodriguez LV, Terris MK. Risks and complications of transrectal ultrasound guided prostate needle biopsy: a prospective study and review of the literature. J Urol 1998; 160: 2115-20.

6 Carlsson S, Aus G, Wessman C, Hugosson J. Anxiety associated with prostate cancer screening with special reference to men with a positive screening test (elevated PSA)—results from a prospective, population-based, randomised study. Eur $J$ Cancer 2007; 43: 2109-16.
7 Carlson GD, Calvanese CB, Partin AW. An algorithm combining age, total prostatespecific antigen (PSA), and percent free PSA to predict prostate cancer: results on 4298 cases. Urology 1998; 52: 455-61.

8 Eastham JA, May R, Robertson JL, Sartor O, Kattan MW et al. Development of a nomogram that predicts the probability of a positive prostate biopsy in men with an abnormal digital rectal examination and a prostate-specific antigen between 0 and 4 ng/mL. Urology 1999; 54: 709-13.

9 Potter SR, Horniger W, Tinzl M, Bartsch G, Partin AW et al. Age, prostate specific antigen, and digital rectal examination as determinants of the probability of having prostate cancer. Urology 2001; 57: 1100-4.

10 Ohori M, Swindle P. Nomograms and instruments for the initial prostate evaluation: the ability to estimate the likelihood of identifying prostate cancer. Semin Urol Oncol 2002; 20: 116-22.

11 Djavan B, Remzi M, Zlotta A, Seitz C, Snow P et al. Novel artificial neural network for early detection of prostate cancer. J Clin Oncol 2002; 20: 921-9.

12 Iasonos A, Schrag D, Raj GV, Panageas KS. How to build and interpret a nomogram for cancer prognosis. J Clin Oncol 2008; 26: 1364-70.

13 Garzotto M, Hudson RG, Peters L, Hsieh YC, Barrera E et al. Predictive modeling for the presence of prostate carcinoma using clinical, laboratory, and ultrasound parameters in patients with prostate specific antigen $<10 \mathrm{ng} / \mathrm{mL}$. Cancer 2003; 98: 1417-22.

14 Lopez-Corona E, Ohori M, Scardino PT, Reuter VE, Gonen M et al. A nomogram for predicting a positive repeat prostate biopsy in patients with a previous negative biopsy session. J Urol 2003; 170: 1184-8.

15 Ouzaid I, Yates DR, Hupertan V, Reuter VE, Gonen M et al. A direct comparison of the diagnostic accuracy of three prostate cancer nomograms designed to predict the likelihood of a positive initial transrectal biopsy. Prostate 2012; 72: 1200-6.

16 Jemal A, Center MM, DeSantis C, Ward EM. Global patterns of cancer incidence and mortality rates and trends. Cancer Epidemiol Biomarkers Prev2010. 19. 1893-907.

17 Kattan MW, Eastham JA, Stapleton AM, Wheeler TM, Scardino PT. A preoperative nomogram for disease recurrence following radical prostatectomy for prostate cancer. J Natl Cancer Inst 1998; 90: 766-71.

18 Tang P, Chen H, Uhlman M, Lin YR, Deng XR et al. A nomogram based on age, prostatespecific antigen level, prostate volume and digital rectal examination for predicting risk of prostate cancer. Asian J Androl 2013; 15: 129-33.

19 Thompson IM, Ankerst DP, Chi C, Goodman PJ, Tangen CM et al. Assessing prostate cancer risk: results from the Prostate Cancer Prevention Trial. J Natl Cancer Inst 2006; 98: 529-34.

20 Hernandez DJ, Han M, Humphreys EB, Mangold LA, Taneja SS et al. Predicting the outcome of prostate biopsy: comparison of a novel logistic regression-based model, the prostate cancer risk calculator, and prostate-specific antigen level alone. BJU Int 2009; 103: 609-14.

21 Park JY, Yoon S, Park MS, Cho DY, Park HS et al. Initial biopsy outcome prediction in Korean patients-comparison of a noble web-based Korean prostate cancer risk calculator versus prostate-specific antigen testing. J Korean Med Sci 2011; 26: 85-91.

22 Chun FK, Karakiewicz PI, Huland $\mathrm{H}$, Graefen M. Role of nomograms for prostate cancer in 2007. World J Urol 2007; 25: 131-42.

23 Benecchi L, Pieri AM, Melissari M, Potenzoni M, Pastizzaro CD et al. A novel nomogram to predict the probability of prostate cancer on repeat biopsy. J Urol 2008; 180: 146-9

24 Lughezzani G, Lazzeri M, Larcher A, Lista G, Scattoni V et al. Development and internal validation of a Prostate Health Index based nomogram for predicting prostate cancer at extended biopsy. J Urol 2012; 188: 1144-50.

25 Shariat SF, Karakiewicz PI, Suardi N, Kattan MW. Comparison of nomograms with other methods for predicting outcomes in prostate cancer: a critical analysis of the literature. Clin Cancer Res 2008; 14: 4400-7. 